\title{
Treatment of Vertically Unstable Sacral Fractures with Pelvic External Fixation and Skeletal Traction
}

\author{
Xueyuan $\mathrm{Wu}^{1^{*}}$, Hongbin $\mathrm{Zhu}^{2}$, Heng $\mathrm{Du}^{{ }^{*}}$, Wei Ma ${ }^{1 \#}$, Chihua Guo ${ }^{1}$ \\ ${ }^{1}$ Department of Orthopaedics, The First Affiliated Hospital, Medical School, Xi'an Jiaotong University, Xi'an, China \\ ${ }^{2}$ Department of Orthopaedics, Xi' an Gaoxin Hospital, Xi'an, China \\ Email: " wologyuan@163.com
}

Received January 16, 2013; revised February 19, 2013; accepted February 28, 2013

Copyright (C) 2013 Xueyuan Wu et al. This is an open access article distributed under the Creative Commons Attribution License, which permits unrestricted use, distribution, and reproduction in any medium, provided the original work is properly cited.

\begin{abstract}
Objective: To determine the efficacy in treatment of vertically unstable sacral fractures with pelvic external fixation and skeletal traction. Patients and Methods: The pelvic external fixation and skeletal traction were applied on 12 cases of vertically unstable sacral fracture (eight males and four females, at age of 19 - 52 years, mean 35.2 years) including two cases of Denis Zone I, nine of Zone II, and one of Zone III. All patients were treated with the pelvic external fixation, and pre- and post-operational longitudinal skeletal traction. Results: The mean operation time, skeletal traction time, skeletal traction removal time and full weight bearing time were $38 \mathrm{~min}, 4.5$ weeks, 8.5 weeks, and 10.3 weeks respectively. All these patients were followed up for 12 - 36 months (average, 22.5 months), which showed no associated complication. The function results were rated as excellent in four cases, good in six and fair in two, with a mean score of 84.4 points, according to Majeed scoring system. Conclusion: The anterior pelvic external fixation with continuous skeletal traction can reliably restore and stabilize the vertically unstable sacrum fracture with excellent functional and radiographic outcomes. It is an ideal method to treat unstably vertical sacral fracture.
\end{abstract}

Keywords: Fracture Fixation; External; Traction; Sacrum

\section{Introduction}

Vertically unstable sacral fractures are mainly caused by high-energy trauma. Most of these fractures are combined with spinal injuries and/or pelvic ring injuries [1], and also associated with lots of vital complications. The complicated configuration of the sacrum and many overlying structures make it difficult to diagnose immediately and treat properly, thus resulting in high morbidity and longterm mortality rates [2]. Reduction and stabilization of unstable sacrum fractures has been a plaguing problem in clinics.

Over the years, it has a great advancement to treat this kind of fractures due to the development of surgical fixation techniques, including percutaneous iliosacral screws [3], posterior transiliac plating [4], lumbopelvic fixation [5] or triangular osteosynthesis [6], and transiliosacral rod [7]. Although these instruments might provide adequate stability, they are difficult techniques with a steep learning curve. The surgeons must understand the complex and variable sacral anatomy. On the other hand, these

*Xueyuan Wu and Heng Du equally contributed to this article.

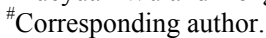

techniques are limited if the patients' vital parameters do not allow definitive operative reduction and fixation.

External fixation is widely recognized as a viable option for unstable pelvic ring disruption with aid of skeletal traction. This method can achieve indirect reduction and high stability, and minimally disrupt soft tissues and rapidly control haemorrhage in emergency room. However, few reports have documented the efficacy of external fixation to treat vertically unstable sacral fractures. In this retrospective study, we evaluated the efficacy in treatment of vertically unstable sacral fractures with pelvic external fixation and skeletal traction.

\section{Patients and Methods}

\subsection{Patients}

Between July 2010 and October 2010, 12 patients with vertically unstable sacral fractures were treated with pelvic external fixation and pre- and post-surgical longitudinal skeletal traction. Of the 12 patients, there were eight males and four females, aged range 19 to 52 years (average age 35.2 years). Of these patients, five cases suffered from traffic accident, four from fall injury and 
the other three from crush injury. Before the operation, the anteroposterior radiographs of the pelvis were performed. If patients were physiologically stable, CT scan was performed to identify the fracture types. These sacral fractures were all vertically unstable. According to Denis' classification [8], two presented the zone I sacral fracture, nine cases the zone II sacral fracture and one case the zone III sacral fracture. At the initial examination, all cases were complicated by multiple fractures, including eight cases suffering disruption of the anterior pelvic ring (two cases with displacement). Five cases were complicated by traumatic shock, three cases by severe posterior soft tissue injuries. Neurological deficits were recorded before operation: two cases had L5 and S1 motor and sensory deficits, two cases had saddle anaesthesia, one case had bowel and bladder dysfunction.

\subsection{Treatment}

After admission into our hospital, all patients were applied transtibial or transcondylar traction, with the initial traction weight of about $1 / 7$ of their body weight. Immediate portable anteroposterior radiographs of the pelvis were reviewed to evaluate reduction accuracy at four hours interval. If the reduction was unsatisfied, $2 \mathrm{~kg}$ weight of traction was added until traction weight of about $1 / 4$ of body weight. The operation was performed immediately, once the displaced fracture was reduced and the pre-operative preparation was completed (Figures 1(a) and (b)).

Under general anaesthesia, the patients were placed supine on a radio-transparent table with continuous longitudinal traction. The bilateral iliac crests were sterilely prepared. The first pin insertion site mounted on iliaccrest was approximately $2 \mathrm{~cm}$ behind the anterior superior iliac spine. A $1 \mathrm{~cm}$ incision was made to access the iliac crest. A drill guide was placed through the incision and down to bone. A $4.5 \mathrm{~mm}$ drill was placed through the guide and advanced approximately $10 \mathrm{~mm}$, taking into account the oblique orientation of the iliac wing. Then a $6.0 \mathrm{~mm}$ pin was screwed at a depth of $4-5 \mathrm{~cm}$. Other two pins were anchored behind the first one at the distance of $2-3 \mathrm{~cm}$ interval. Similarly, three more pins were placed in the contralateral iliac crest. The remaining components of external fixator were then installed on the pins. The fracture was then corrected by external manipulation under fluoroscopy (Figure 1(c)).

The patients with neurologic deficits and $\mathrm{CT}$ evidence of nerve entrapment by the bone flap needed the posterior surgical decompression and fixation, thus they were excluded in our study.

\subsection{Postoperative Management}

On the first postoperative day, pharmacological prophy- laxis and intermittent pneumatic compression were applied to prevent venous thromboembolism (VTE) and pulmonary thromboembolism (PTE). The traction was applied for $4-6$ weeks to maintain the position of the fracture. The external fixator was removed at the sixth to tenth week according to the type and severity of injury. They were permitted to walk with crutches without weight bearing or with partial weight-bearing. Three months later, they were encouraged to walk gradually in specialized rehabilitation department.

\subsection{Follow-Up and Evaluation}

Functional outcome was evaluated according to scoring system proposed by Majeed [9], which was divided into seven factors such as pain (30 points), work (20 points), sitting (10 points), sexual intercourse (4 points), standing (36 points, including walking aid, gait unaided and walking distance). The total score then gave a clinical grade as excellent ( $\geq 85$ points), good (70 - 84 points), fair (55 69 points) or poor ( $<55$ points). The anteroposterior radiological examination of the pelvis was reviewed to evaluate the reduction of the fracture. The largest posterior displacement were recorded and graded according to the method proposed by Tornetta and Matta [10]. Displacement less than $5 \mathrm{~mm}$ was graded as excellent, 5 - 10 $\mathrm{mm}$ as good, $10-20 \mathrm{~mm}$ as fair, and more than $20 \mathrm{~mm}$ as poor. Statistical analyses were performed using SPSS for Windows 15.0.

\section{Results}

The mean time from admission to our hospital to external fixation surgery was $(2.5 \pm 1.5)$ days. The mean operation time for applying the external fixation was $(38 \pm 12)$ min. The blood loss was rare. The bone traction was kept for average $(4.5 \pm 1.7)$ weeks. The external fixation was kept in place for an average duration of $(8.5 \pm 2.4)$ weeks. The mean time to full weight bearing was $(10.3 \pm 3.4)$ weeks, which was dependent on the associated injuries. The patients were all followed up for 12 - 36 months (average, 22.5 months). There was no pin track infection. Secondary loss of reduction or external fixation loosening or breakage did not occur. There was no obvious lower limb length inequality. All the sacral fractures healed eventually Figure 1(d). Radiographically, six of the patients were graded as excellent, four good, and two fair. No patients were graded as poor. All patients completed the Majeed functional investigation at the final follow-up. The mean score was 84.4 points (range, 49 - 98 points), which were graded as four excellent, six good, two fair. Of five patients with neurological deficit before operation, four had complete recovery, and one had partial neurological impairment of L5 at the latest re-evaluation. 


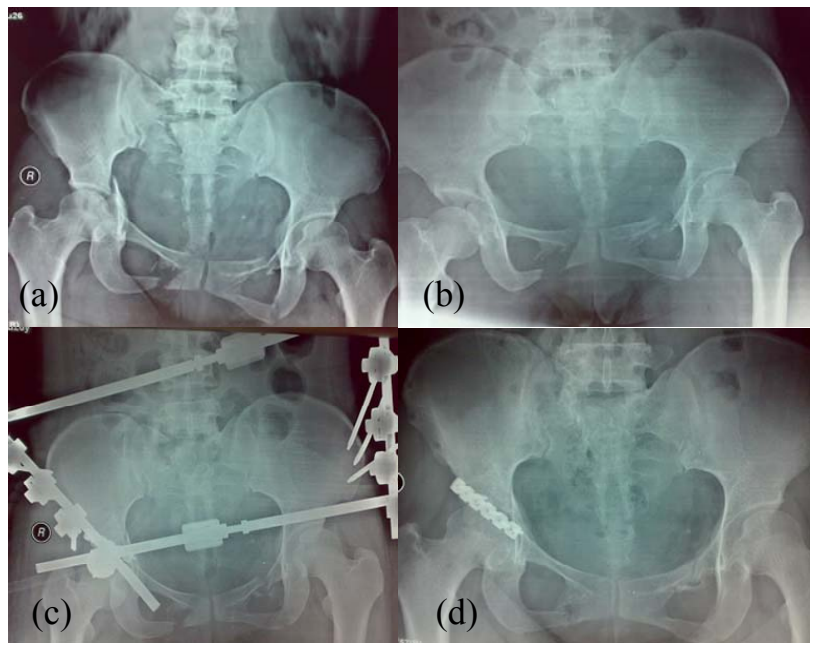

Figures 1. A 26-year-old woman who sustained right Denis II sacral fractures by a car accident. (a) Preoperative X-ray film; (b) Preoperative $X$-ray film of reduction by skeletal traction; (c) Postoperative X-ray film (satisfactory fracture reduction); (d) Postoperative $\mathrm{X}$-ray at 2 years after the operation.

\section{Discussion}

The proper treatment of sacral fractures poses significant challenges for orthopaedic surgeons. It is widely recognized that the treatment goals for sacral trauma are to reduce and stabilize the unstable sacrum fracture, protect the neurological structures, decompress the neural elements and prevent the frequent complications [4]. Over the years, a variety of instrumentations for reduction and stabilization of sacral fracture have been developed, such as transiliac screws and plates, transiliac rods, lumboiliac plates, and iliosacral screws. Some of these fixation techniques can apply sufficiently rigid fixation and gain good results by direct reduction of fracture. However, these conventional posterior operation approaches have such disadvantages as long incisions, long exposure time, soft tissue complications and more blood loss [11]. For example, infection rates have been reported to $27 \%$ when using a posterior approach, due to the presence of soft tissue injuries around the pelvis represented by the MorelLavallee-lesion [4]. Bellabarba et al. [12] retrospectively evaluated the rate of complications of 19 consecutive patients treated with rigid segmental lumbopelvic stabilization. Major complications were primarily related connecting rods in 6/19 patients $(31 \%)$ and wound healing disturbances in $5 / 19$ (26\%). The minimally invasive technique such as percutaneous transsacral screw fixation provides shorter operation time and less blood loss compared with an open method for treating sacral fractures [13]. However, it is a difficult technique, with a steep learning curve, and the surgeon must be familiar with the complex and variable sacral anatomy [14]

\subsection{Stabilization}

External fixation is widely used in treatment of unstable anterior pelvic fractures in the initial stabilization of a hemodynamically unstable patient [15]. It also has a definite role to maintain reduction and finally stabilize pelvic injuries [16]. Safety and simplicity are the two major advantages in contrast to internal fixation [17]. However, there are few clinical reports and biomechanical analysis concerning treatment of sacral fracture by external fixation. Gunterberg et al. [18] carried out an experimental study on the loading of unilateral fractures of the sacrum and sacro-iliac dislocations with a trapezoid external compression fixation frame. The results indicated this frame properly reduced and stabilized these injuries. Meanwhile, it also gave sufficient stability to allow weightbearing in the upright standing position. Gardner et al. [19] confirmed that pelvic external fixation pins which were placed in the supraacetabular region imparted enough compression forces across the sacroiliac joint. Kim et al. [20] measured the stability of anterosuperior locations of pins into the iliac crest in pelvic injuries. They found that the external fixation could significantly reduce sacroiliac joint separation. Ilizarov external fixation device was also reported to efficiently treat unstable fracture of the posterior pelvic ring [21]. Based on these researches, it provides convincing evidence for the surgeons to stabilize vertical sacral fracture using anterior pelvic external fixation. In our consecutive series of 12 patients, the indirect reduction and stabilization were achieved by pelvic external fixation. At the final radiographical follow-up, ten patients were graded as excellent and good, and two fair. Our study confirmed that the application of external fixation was able to reliably maintain the stabilization of sacral fracture.

In the application of this technique, we have not used the technique of pins insertion into supraacetabular region in an anterior to posterior direction described by Gardner et al. [19], although it provides more compression [20]. Instead, we have performed pelvic external fixation perpendicularly anchoring these pins into each iliac crest in a superior to inferior direction, which has been used most frequently. Because former technique has potential disadvantages related to interference with the hip flexion, risk of hip joint penetration and damage to the lateral femoral cutaneous nerve branches [22,23].

\subsection{Function Reduction}

It is important to restore the anatomic alignment of scrum fracture. However, it is a formidable task even by open reduction from posterior surgical approach [24]. Therefore, a controversial topic has arisen concerning whether 
anatomical reduction outcome is superior to functional reduction outcome [25]. Gansslen et al. [26] recommended anatomical reduction and decompression for the instable sacral fracture has instability. Many authors reported the indirect reduction could achieve the same excellent function result as the anatomical reduction $[13$, 27]. Chen et al. [11] reported excellence rate up to $85.7 \%$ according to the Majeed scoring system using indirect reduction and percutaneous internal fixation. In our study, complete or essential reduction of displaced fractures had been achieved. In the application of indirect reduction, a reasonable and large-weight longitudinal skeletal traction prior to operation should be administered to reduce the vertical displacement. The patients can endure traction weight of about $1 / 7-1 / 4$ of their body weight, if the weight is added gradually. In our experience, we usually add $2 \mathrm{~kg}$ at four hours intervals. Repeated radiographic films are taken to make sure the extent of deformity correction. Postoperatively, the consistent traction is kept to maintain the reduction for average $4-6$ weeks. Some authors advocated that pelvic external fixation combined with longitudinal traction badly affected the earlier weight-bearing and returned to their previous activity and work. However, it is not possible for patients with unstable sacral fractures, because they are frequently suffered from multiple fractures and neurological injuries [4]. With this method, all patients showed union of the sacral fracture and a satisfactorily stable pelvis. No secondary loss of reduction occurred. The patients had satisfactory functional results. Therefore, we made the primary conclusion that the iliac wing as a fixation site for lumbopelvic stabilizations in the pelvis and the pre- and post-operative continuous skeletal traction can restore and maintain the sacral fracture, and gain a good outcome to treat vertically unstable sacrum fracture.

As for neurological compromise, another controversial topic is whether surgical decompression of the neural elements improves better recovery results than conservative treatment $[2,5]$. Both methods have their supporters, but there is lack of solid evidence. Until recently, there have been no definite methods to accurately prove if the nerve roots are anatomical continuity. Therefore, many authors have suggested, only decompression of nerve roots is involved if debris or fragments compressed neural roots identified by CT scanning $[4,11,28]$. We support this viewpoint, so the sacral fractures in this case are excluded in our study and submitted to posterior operation. In our study, five of our patients were diagnosed with neurological deficit before operation. Only one did not completely recovered.

\subsection{Advantages}

The major advantage of this method to treat fracture is in accordance to BO biomechanical conception. Potential benefits include minimizing soft tissue damage, shortening operation time, reducing perioperative complications as well as gaining faster rehabilitation compared with posterior open operation. In our research, the mean operative time was $38 \mathrm{~min}$ and the blood loss was rare. The closed reduction minimally interferes with the posterior soft tissue, hence avoiding the risk of infection. The major complication is pin sites infection, which can be eliminated by careful nursing and cleansing of the bases of the pins with dilute hydrogen peroxide twice daily. All 12 patients in our study had no pins track infection. Another vigilant complication is the development of VTE and PTE. Niikura et al. [29] reported that 19 patients $(41.3 \%)$ were diagnosed with VTE, and PTE in ten $(21.7 \%)$ in pelvic and acetabular fractures. The incidences were significantly higher compared with trauma patients without pelvic and acetabular fractures. Fortunately, all were asymptomatic. Administration of routine thromboprophy-laxis is fully justified [30]. In our study, pharmacological prophylaxis and intermittent pneumatic compression were applied to prevent venous thromboembolism. There was no form of symptomatic VTE and PTE.

\subsection{Limitations}

This approach has several limitations. Pelvic external fixation has no effect on the transverse sacral fractures, though they constitute only $3 \%$ to $5 \%$ of all sacral fractures [31]. It also has limited function on the recovery of neurological compromise caused by compression of bone fragment, which we mentioned previously. The use of anterior external fixation should be under the circumstances of intact or the restoration of intact anterior pelvic ring. In this case, the incision of anterior operative approach and external pin sites will be conflicted. Despite the existence of these drawbacks, the anterior pelvic external fixation with skeletal traction is still an ideal surgical approach to treat vertically unstable sacral fractures.

Because this type of injury is relatively rare, our study represents a small cohort of patients. We are unable to compare the method of external fixation with the various operation methods via posterior approach by prospective and/or randomized studies. We also cannot draw the firm conclusion that external fixation with skeletal traction superior to another treatment method. However, we should not neglect its advantages regarding safety, efficacy and low complication.

\section{Conclusion}

The anterior pelvic external fixation mounted in iliac crest and the pre- and post-operative continuous skeletal traction can restore and maintain the vertically unstable 
sacrum fracture. It achieves excellent function and radiographic outcome. Meanwhile, it has such advantages as simple manipulation and fewer complications. It is an ideal surgical approach to treat unstably vertical sacral fractures.

\section{REFERENCES}

[1] C. Lee, Y. S. Park, S. W. Baek, H. S. Kim and K. C. Park, "Management of Sacral Fractures Associated with Spinal or Pelvic Ring Injury," The Journal of Trauma and Acute Care Surgery, Vol. 73, No. 1, 2012, pp. 239-242. doi:10.1097/TA.0b013e31825a79d2

[2] V. A. Lykomitros, K. A. Papavasiliou, Z. M. Alzeer, F. E. Sayegh, J. M. Kirkos and G. A. Kapetanos, "Management of Traumatic Sacral Fractures: A Retrospective Case-Series Study and Review of the Literature," Injury, Vol. 41, No. 3, 2010, pp. 266-272. doi:10.1016/j.injury.2009.09.008

[3] G. Osterhoff, C. Ossendorf, G. A. Wanner, H. P. Simmen and C. M. Werner, "Posterior Screw Fixation in Rotationally Unstable Pelvic Ring Injuries," Injury, Vol. 42, No. 10, 2011, pp. 992-996. doi:10.1016/j.injury.2011.04.005

[4] T. Suzuki, D. J. Hak, B. H. Ziran, S. A. Adams, P. F. Stahel, S. J. Morgan and W. R. Smith, "Outcome and Complications of Posterior Transiliac Plating for Vertically Unstable Sacral Fractures," Injury, Vol. 40, No. 4, 2009, pp. 405-409. doi:10.1016/j.injury.2008.06.039

[5] G. Q. Tan, J. L. He, B. S. Fu, L. X. Li, B. M. Wang and D. S. Zhou, "Lumbopelvic Fixation for Multiplanar Sacral Fractures with Spinopelvic Instability," Injury, Vol. 43, No. 8, 2012, pp. 1318-1325. doi:10.1016/j.injury.2012.05.003

[6] H. C. Sagi, "Technical Aspects and Recommended Treatment Algorithms in Triangular Osteosynthesis and Spinopelvic Fixation for Vertical Shear Transforaminal Sacral Fractures," Journal of Orthopaedic Trauma, Vol. 23, No. 5, 2009, pp. 354-360. doi:10.1097/BOT.0b013e3181a1143a

[7] D. M. Sciubba, J. P. Wolinsky, K. D. Than, Z. L. Gokaslan, T. F. Witham and K. P. Murphy, "CT Fluoroscopically Guided Percutaneous Placement of Transiliosacral Rod for Sacral Insufficiency Fracture: Case Report and Technique," American Journal of Neuroradiology, Vol. 28, No. 8, 2007, pp. 1451-1454. doi:10.3174/ajnr.A0665

[8] F. Denis, S. Davis and T. Comfort, "Sacral Fractures: An Important Problem. Retrospective Analysis of 236 Cases," Clinical Orthopaedics and Related Research, Vol. 227, 1988, pp. 67-81.

[9] S. A. Majeed, "Grading the Outcome of Pelvic Fractures," Journal of Bone and Joint Surgery, Vol. 71, No. 2, 1989, pp. 304-306.

[10] P. R. Tornetta and J. M. Matta, "Outcome of Operatively Treated Unstable Posterior Pelvic Ring Disruptions," Clinical Orthopaedics and Related Research, Vol. 329, 1996, pp. 186-193. doi:10.1097/00003086-199608000-00022

[11] H. W. Chen, G. D. Liu, S. Ou, G. S. Zhao and J. Pan,
"Treatment of Unstable Sacral Fractures with Percutaneous Reconstruction Plate Internal Fixation," Acta Cirúrgica Brasileira, Vol. 27, No. 5, 2012, pp. 338-342. doi:10.1590/S0102-86502012000500010

[12] C. Bellabarba, T. A. Schildhauer, A. R. Vaccaro and J. R. Chapman, "Complications Associated with Surgical Stabilization of High-Grade Sacral Fracture Dislocations with Spino-Pelvic Instability," Spine, Vol. 31, No. 11, 2006, pp. S80-S88.

[13] M. A. Konig, U. Seidel, P. Heini, R. Orler, N. A. Quraishi, A. A. Boszczyk and B. M. Boszczyk, "Minimal-Invasive Percutaneous Reduction and Transsacral Screw Fixation for U-shaped Fractures," Journal of Spinal Disorders \& Techniques, Vol. 26, No. 1, 2011, pp. 48-54. doi:10.1097/BSD.0b013e3182318539

[14] M. Rysavy, T. Pavelka, M. Khayarin and V. Dzupa, "Iliosacral Screw Fixation of the Unstable Pelvic Ring Injuries," Acta Chirurgiae Orthopaedicae et Traumatologiae Cechoslovaca, Vol. 77, No. 3, 2010, pp. 209-214.

[15] S. B. Hu, H. Xu, H. B. Guo, T. Sun and C. J. Wang, "External Fixation in Early Treatment of Unstable Pelvic Fractures," Chinese Medical Journal (English Edition), Vol. 125, No. 8, 2012, pp. 1420-1424.

[16] P. Grubor, S. Milicevic, M. Biscevic and R. Tanjga, "Selection of Treatment Method for Pelvic Ring Fractures," Medical Archives, Vol. 65, No. 5, 2011, pp. 278-282.

[17] S. A. Majeed, "External Fixation of the Injured Pelvis. The Functional Outcome," The Journal of Bone \& Joint Surgery, Vol. 72, No. 4, 1990, pp. 612-614.

[18] B. Gunterberg, I. Goldie and P. Slatis, "Fixation of Pelvic Fractures and Dislocations. An Experimental Study on the Loading of Pelvic Fractures and Sacro-Iliac Dislocations after External Compression Fixation," Acta Orthopaedica Scandinavica, Vol. 49, No. 3, 1978, pp. 278-286. doi:10.3109/17453677809005765

[19] M. J. Gardner, D. Kendoff, S. Ostermeier, M. Citak, T. Hufner, C. Krettek and S. E. Nork, "Sacroiliac Joint Compression Using an Anterior Pelvic Compressor: A Mechanical Study in Synthetic Bone," Journal of Orthopaedic Trauma, Vol. 21, No. 7, 2007, pp. 435-441. doi:10.1097/BOT.0b013e318126bb8e

[20] W. Y. Kim, T. C. Hearn, O. Seleem, E. Mahalingam, D. Stephen and M. Tile, "Effect of Pin Location on Stability of Pelvic External Fixation," Clinical Orthopaedics and Related Research, Vol. 316, 1999, pp. 237-244. doi:10.1097/00003086-199904000-00030

[21] S. M. Tomic and N. S. Slavkovic, "Unstable Fractures of the Pelvis Treated by Ilizarov External Fixation Device," Acta Chirurgica Iugoslavica, Vol. 57, No. 1, 2010, pp. 25-29. doi:10.2298/ACI1001025T

[22] L. B. Solomon, A. P. Pohl, M. J. Chehade, A. M. Malcolm, D. W. Howie and M. Henneberg, "Surgical Anatomy for Pelvic External Fixation," Clinical Anatomy, Vol. 21, No. 7, 2008, pp. 674-682. doi:10.1002/ca.20697

[23] M. J. Gardner and S. E. Nork, "Stabilization of Unstable Pelvic Fractures with Supraacetabular Compression External Fixation," Journal of Orthopaedic Trauma, Vol. 21, No. 4, 2007, pp. 269-273. doi:10.1097/BOT.0b013e318030e3e4 
[24] T. Taguchi, S. Kawai, K. Kaneko and D. Yugue, "Operative Management of Displaced Fractures of the Sacrum," Journal of Orthopaedic Science, Vol. 4, No. 5, 1999, pp. 347-352. doi:10.1007/s007760050115

[25] D. C. Templeman, T. Simpson and J. M. Matta, "Surgical Management of Pelvic Ring Injuries," Instructional Course Lectures, Vol. 54, 2005, pp. 395-400.

[26] A. Gansslen, H. C. Pape, U. Lehmann, U. Lange, C. Krettek and T. Pohlemann, "Open Reduction and Internal Fixation of Unstable Sacral Fractures," Zentralblatt für Chirurgie, Vol. 128, No. 1, 2003, pp. 40-45.

[27] D. Schweitzer, A. Zylberberg, M. Cordova and J. Gonzalez, "Closed Reduction and Iliosacral Percutaneous Fixation of Unstable Pelvic Ring Fractures," Injury, Vol. 39, No. 8, 2008, pp. 869-874.

[28] M. A. Ayoub, "Vertically Unstable Sacral Fractures with Neurological Insult: Outcomes of Surgical Decompression and Reconstruction Plate Internal Fixation," Interna- tional Orthopaedics, Vol. 33, No. 1, 2009, pp. 261-267. doi:10.1007/s00264-007-0468-9

[29] T. Niikura, S. Y. Lee, K. Oe, A. Koh, T. Koga, Y. Dogaki, E. Okumachi and M. Kurosaka, "Incidence of Venous thromboembolism in Pelvic and Acetabular Fractures in the Japanese Population," Journal of Orthopaedic Science, Vol. 17, No. 3, 2012, pp. 233-238. doi:10.1007/s00776-012-0203-2

[30] R. K. Sen, A. Kumar, S. K. Tripathy, S. Aggarwal, N. Khandelwal and S. R. Manoharan, "Risk of Postoperative Venous Thromboembolism in Indian Patients Sustaining Pelvi-Acetabular Injury," International Orthopaedics, Vol. 35, No. 7, 2011, pp. 1057-1063. doi:10.1007/s00264-010-1093-6

[31] L. A. Robles, "Transverse Sacral Fractures," Spine Journal, Vol. 9, No. 1, 2009, pp. 60-69. doi:10.1016/j.spinee.2007.08.006 